\title{
Openness in Öffentlichen Bibliotheken - beschleunigt durch Erfahrungen in der Pandemie?
}

\author{
Tina Grahl ${ }^{1}$ und Stephan Wünsche ${ }^{2}$ \\ ${ }^{1}$ Bibliothek der Technischen Hochschule Aschaffenburg, \\ https://orcid.org/0000-0002-4974-708X \\ ${ }^{2}$ Universitätsbibliothek Leipzig, \\ https://orcid.org/0000-0001-9552-4402
}

Bibliotheken sind Orte der sozialen Teilhabe und Emanzipation, der Demokratieförderung und Bildung. Sie dienen als Treffpunkte für den intellektuellen und kulturellen Austausch. Dieses Selbstverständnis, das besonders für Öffentliche Bibliotheken eine zentrale Rolle spielt, auch in die Tat umzusetzen, wurde durch die Corona-Pandemie jedoch so schwierig wie vielleicht nie zuvor. Die Herausforderung besteht darin, die Gesundheit der Nutzer*innen und Mitarbeiter*innen zu schützen, ohne den eigenen Daseinszweck, die eigene Mission aufzugeben. So zwingt die Pandemie Bibliotheken zu agilem Handeln und Innovationen. Sie reagieren auf verordnete Schließungen mit Abholservice und Lieferdiensten, rücken digitale Medien in den Fokus und organisieren digitale Lesungen und Online-Angebote zur Leseförderung. Bibliotheken öffnen sich auf neue Weise, um ihre Nutzer*innen weiterhin zu erreichen. Die Corona-Pandemie hat insofern auch positive Effekte: Öffentliche Bibliotheken können mit neuen oder erweiterten Services dauerhaft sichtbarer werden. Deutlich wird in der Pandemie vor allem, wie viel Öffentliche Bibliotheken im Bereich Openness leisten.

Unter Openness (engl. für Offenheit) wird der Abbau von Barrieren, die Förderung von Teilhabe und Transparenz sowie der Einsatz für offenen Zugang zu Wissen und Information verstanden. Um die Openness der Bibliothek in der eigenen Einrichtung, der Bibliothekscommunity und für Nutzer*innen sichtbarer zu machen, wurde 2016 der Open Library Badge (OLB) entwickelt. Der Open Library Badge ist ein virtuelles Abzeichen für Bibliotheken, die sich für Openness einsetzen. Er umfasst aktuell 15 Kriterien und damit 15 konkrete Möglichkeiten, wie Bibliotheken dieses Ideal in die Praxis umsetzen können. Erfüllen Bibliotheken mindestens fünf davon, können sie sich um den Badge bewerben. Eine Jury prüft die Bewerbung und vergibt dann die Auszeichnung.

Die Trägerinnen des Open Library Badge 2016 waren allesamt Wissenschaftliche Bibliotheken. Das lag zum einen daran, dass es zu dieser Zeit vor allem Mitarbeiter*innen Wissenschaftlicher Bibliotheken waren, die die fachliche Diskussion um Openness in Bibliotheken vorantrieben. Zum anderen - und damit zusammenhängend - griffen die damaligen BadgeKriterien vorrangig Schwerpunkte Wissenschaftlicher Bibliotheken wie das Open-AccessPublizieren oder Text- und Data-Mining auf. Als im Jahr 2020 die Kriterien überarbeitet und erweitert wurden, war es das erklärte Ziel des OLB-Teams, bei der Formulierung der Kriterien auch Öffentliche Bibliotheken mitzudenken und zur Bewerbung zu ermuntern. 
Im November 2020 wurde die Stadtbibliothek Erlangen als erste Öffentliche Bibliothek mit dem Open Library Badge ausgezeichnet. Dieser Erfolg zeigt, dass eine Bewerbung um den Open Library Badge für Öffentliche Bibliotheken umsetzbar und lohnenswert ist.

\section{Attraktive Kriterien für Öffentliche Bibliotheken}

Während einige Kriterien nach wie vor auf Wissenschaftliche Bibliotheken ausgerichtet sind, gibt es in der aktuellen Version des Open Library Badge nun auch neun Kriterien, die von vielen Öffentlichen Bibliotheken bereits umgesetzt werden oder über deren Umsetzung nachzudenken sich für diese lohnt. Um den Open Library Badge zu erhalten, müssen nur fünf davon erfüllt werden.

Im Folgenden haben wir Beispiele aufgelistet, wie Öffentliche Bibliotheken Openness umsetzen können. Die vollständige Beschreibung aller Kriterien des Open Library Badge und der geforderten Nachweise finden sich auf der OLB-Homepage.

\section{Bedienbarkeit für alle Menschen}

Die Homepage der Bibliothek ist barrierefrei bzw. barrierearm. Das heißt, sie weist im OnlineSchnelltest WAVE keine Fehler auf bzw. die aufgetretenen Fehler lassen sich z. B. durch Corporate-Design-Vorgaben einer übergeordneten Institution begründen. Eine Selbstbewertung nach BITV/WCAG ergibt, dass jeder Prüfschritt mindestens ,eher erfüllt““ ist.

\section{Fortbildungsangebote}

Die Bibliothek hat eine Fortbildung (mit-)organisiert, die ein Openness-Thema im weitesten Sinne behandelt - bspw. die Umsetzung einer barriearmen Homepage oder eines Flyers in Einfacher Sprache, eine digitale Austauschrunde zu den Best Practices während der Pandemie oder zur Organisation eines Wiki-Editathons. Dazu hat sie auch externe Personen wie Mitarbeiter*innen anderer Bibliotheken, Lehrer*innen, Mitarbeiter*innen aus Kitas, Ehrenamtliche aus Vereinen, Mitarbeiter*innen der Stadtverwaltung etc. explizit eingeladen. Eine Ankündigung oder ein Bericht im Nachgang genügt als Nachweis.

\section{Lehr- und Lernmaterialien unter offener Lizenz}

Schulende nutzen Präsentationen oder Handouts z. B. für Recherche-Workshops mit Schüler*innen oder (digitale) Schnitzeljagden für Grundschüler*innen. Wenn die Schulenden diese Materialien auf der Bibliothekshomepage oder etwa bei Zenodo unter offener Lizenz (z. B. CC-BY) veröffentlichen, können sie durch andere Bibliotheken nachgenutzt, angepasst und weiterentwickelt werden.

\section{Einbindung eigener und externer Nutzer*innen}

Die Bibliothek hat in den letzten zwei Jahren Nutzer*innen eingebunden, indem sie zum Beispiel eine Umfrage zu deren Erwartungen oder zur Zufriedenheit mit Serviceangeboten durchgeführt hat. Egal ob eine große Umfrage in der Stadt oder eine einfache FlipchartUmfrage in der Bibliothek zur Zufriedenheit mit einem bestimmten Raumbereich in der Bibliothek oder einem konkreten Teil des Bestandes. Ein Bericht auf der Homepage, im Blog oder in der regionalen Zeitung über die Aktion genügt als Nachweis. 


\section{Geschäftsgänge veröffentlichen}

Die Bibliothek hat einen erprobten Workflow für einen Arbeitsbereich, z. B. die Planung von Veranstaltungen und Lesungen oder die Einrichtung eines Library Escape Rooms, der sich mit wenig Mühe aufbereiten und verschriftlichen lässt, sodass er für andere nachnutzbar ist. Für eine Veröffentlichung bieten sich Zenodo, die Bibliothekshomepage oder der Blog der Bibliothek an.

\section{Offene Wissenscommunities unterstützen}

Mitarbeiter*innen der Bibliothek unterstützen offene Wissenscommunities. Das kann etwa durch gemeinsam organisierte Veranstaltungen oder die Bereitstellung von WLAN und Räumlichkeiten geschehen. Zu offenen Wissenscommunities zählen beispielsweise Maker-SpaceVereine, Repair-Cafés, der Chaos Computer Club oder die örtliche Wikipedia-Community. Auch die Beteiligung an der Aktion \#1Lib1Ref durch Bibliotheksmitarbeiter*innen ist möglich. Als Nachweis berichtet die Bibliothek auf der Homepage, in der regionalen Zeitung oder im Blog darüber.

\section{Selbst nur Open Access publizieren}

Bibliotheksmitarbeiter*innen haben in den vergangenen zwölf Monaten ausschließlich Open Access publiziert, sodass ein dauerhafter offener Zugang zur Publikation gewährleistet ist. Falls die Bibliothek das Kriterium noch nicht erfüllt: Wie wäre es mit einem Erfahrungsbericht in der Reihe „Corona-Splitter“ des Magazins 027.7?

\section{Fotos der Bibliothek nachnutzbar machen}

Viele Öffentliche Bibliotheken sind mittlerweile auf Instagram aktiv. Leider sind diese Fotos nicht durch andere Personen oder Einrichtungen nachnutzbar. Fotos können stattdessen oder auch parallel auf Wikimedia Commons oder auf der Plattform Flickr unter einer offenen Lizenz (z. B. CC0 oder CC-BY) zur Verfügung gestellt werden. So können andere die Fotos nachnutzen (im Falle von CC-BY unter Angabe des Urhebers, d. h. der Bibliothek). Dies ermöglicht eine urheberrechtskonforme Verwendung z. B. durch Schüler*innen, Studierende und Lehrende in Referaten und Präsentationen.

\section{Bibliothek als offener Ort}

Die Bibliothek bietet Veranstaltungen mit integrativer, inklusiver und/oder emanzipativer Ausrichtung an. Dazu zählen Veranstaltungen zur Leseförderung wie mehrsprachiges Vorlesen, Bilderbuchkino oder Lesepaten-Projekte sowie Veranstaltungen im Bereich Maker Spaces, Demokratieförderung oder Medienkompetenz (Fake News u. ä.). Auch spezielle Angebote für Migrant*innen und Geflüchtete oder Senior*innen fallen unter inklusive Angebote. Ein Link zur Beschreibung der Veranstaltung oder zum Bericht auf der Homepage oder im Bibliotheksblog ist als Nachweis ausreichend.

Die weiteren sechs Kriterien des Open Library Badge sind: Open-Source-Software mitgestalten, Openness in der Personalentwicklung fördern, Text- und Data-Mining ermöglichen, Kostentransparenz beim Erwerb herstellen, Open-Access-Ressourcen sichtbar machen und Open-Access-Potenziale aufdecken. 


\section{Bewerbung}

Sinnvollerweise starten Bibliotheken in den Bewerbungsprozess, indem sie prüfen, welche der 15 Kriterien sie bereits mit den geforderten Nachweisen erfüllen. Zur Orientierung eignen sich auch die auf der Homepage der Initiative gelisteten Best-Practice-Beispiele der bisherigen Badge-Trägerinnen. Haben sie bereits fünf Kriterien erfüllt, können sie sich direkt um den Badge bewerben. Fehlen noch Nachweise, lohnt es sich zu prüfen, welche Kriterien mit wenig Aufwand oder kleinen Nachbesserungen erfüllt werden können. Ziel des Badges ist es auch, dass Bibliotheken ihren Fokus innerhalb des Bewerbungsprozesses auf neue Möglichkeiten lenken, bestehende Services ausbauen und möglicherweise neue Angebote entwickeln. Bibliotheken können die Bewerbung daher im Sinne der Open Innovation nutzen und die Kriterien und Nachweise im gesamten Team, d. h. mit Bibliothekar*innen, FaMIs, Ehrenamtlichen und allen weiteren Teammitgliedern diskutieren. So kommen verschiedene Blicke auf das Thema zusammen.

Die Bewerbung erfolgt über ein Internetformular auf der Seite der Initiative. Die Nachweise zu den erfüllten Kriterien können als Links - etwa zur eigenen Homepage, zum Blog oder zu einem Bericht in der Tageszeitung - eingereicht werden. Die Bewerbung wird anschließend durch das Team des Open Library Badge geprüft. Nach erfolgreicher Vergabe kann die Bibliothek den virtuellen Badge auf ihrer Homepage oder in Social-Media-Kanäle einbinden und damit ihre Verdienste sichtbar machen. Außerdem wird die Bibliothek auf der Homepage der Initiative unter den Badge-Trägerinnen gelistet; die erfüllten Kriterien werden unter den Best-Practice-Beispielen ergänzt. Diese dienen wiederum weiteren Bibliotheken als Anregung.

Das OLB-Team ist ein informeller Zusammenschluss von Freiwilligen aus der Bibliotheksbranche. Weil der Badge ursprünglich auf die Aktivitäten Wissenschaftlicher Bibliotheken zurückgeht, stammen die aktuellen Mitglieder vorrangig aus diesem Sektor. Das Team freut sich jederzeit über neue Mitglieder, ausdrücklich auch aus Öffentlichen Bibliotheken. 